\title{
Spectroscopic Analysis and Study of Charge Transport Properties for Pinacyanol Chloride-Organic Acceptor Complex as Potential Optoelectronics Material
}

\author{
Sagarkumar M. Agravat ${ }^{*}$, Vishal R. Jain², Ajay T. Oza² \\ ${ }^{1}$ Gujarat Energy Research \& Management Institute (GERMI), Gandhinagar, India \\ ${ }^{2}$ Department of Physics, S.P. University, Vallabh Vidyanagar, India \\ Email: 'sagravat@gmail.com
}

Received 30 April 2015; accepted 27 July 2015; published 30 July 2015

Copyright (C) 2015 by authors and Scientific Research Publishing Inc.

This work is licensed under the Creative Commons Attribution International License (CC BY).

http://creativecommons.org/licenses/by/4.0/

(c) †) Open Access

\begin{abstract}
Organic photoconductor, pinacyanol chloride, has been studied with infrared spectroscopy because of its thermal activation energy $\left(E_{a}\right)$ and band gap $\left(E_{g}=2 E_{a}\right)$ lying in the infrared range. Particularly, pinacyanol chloride and its charge transfer (CT) complexes with chloranil, DDQ, TCNQ and TCNE as organic acceptors are studied in details. The CT complexes are having neither two absorption edges like ternary complex having one donor and two acceptors nor binary type with Lorentzian or Gaussian envelopes. The forbidden gap is direct band gap except chloranil complex due to increase in molecular distance and CT interaction. There is imperfect nesting and partial screening determining the mid-IR envelope, which is qualitatively different from the envelopes in binary systems. There is inverted parabola in some range below this envelope. It is explained how infrared absorption is related with the applications of such organic photoconductors in optoelectronic devices.
\end{abstract}

\section{Keywords}

Organic Photoconductors, Charge Transfer Complex, Pinacyanol Chloride, Spectroscopy, Optoelectronic Devices, Diodes, LEDs, Solar Cells

\footnotetext{
${ }^{*}$ Corresponding author.

How to cite this paper: Agravat, S.M., Jain, V.R. and Oza, A.T. (2015) Spectroscopic Analysis and Study of Charge Transport Properties for Pinacyanol Chloride-Organic Acceptor Complex as Potential Optoelectronics Material. American Journal of Analytical Chemistry, 6, 694-707. http://dx.doi.org/10.4236/ajac.2015.68067
} 


\section{Introduction}

The subject of charge transfer complexes has been very old [1]-[3]. After finding organic metals and other quasione-dimensional conductors [4] [5], this important branch of chemical physics has undergone a renewed interest. To some of the experimental scientists and applied physicists, it is known as molecular electronics. The use of CT complexes involving both organic and organometallic conductors in dry cells (batteries), p-n junction diodes, FETs, Schottkey diodes and optoelectronic devices like photodiodes, phototransistors, infrared detectors, solar cells, organic LEDs, etc. has been attempted. This is how the subject of one-dimensional conductors has been connected with advanced energetics [6]-[20].

In the present work, we have reported transport properties and effect on band-gap of pinacyanol Chloride when it forms CT with organic acceptors. Transmission spectra of the charge transfer complexes of pinacyanol is reported. The structures of pinacyanol chloride and organic acceptors used in the present work are shown (Figure 1).

\section{Experimental Details}

Pinacyanol chloride - a pure spectroscopy grade was obtained from Aldrich chemical company. It was divided in four equal parts and each part was mixed with similarly pure organic acceptors, namely chloranil, DDQ (2,3dichloro-5,6-dicyano-p-benzoquinone), TCNE (tetracyano ethylene) and TCNQ (7,7,8,8-tetracyano-p-quinodimethane) in 1:1 molecular proportions and grinding was carried for each mixture in a black agate mortar with black agate pestle till the color deepened or changed remarkably.

The charge transfer complexes prepared as above were mixed with dry spectro-grade KBr powder in 5:95 percentage proportions. The $95 \%$ of the $\mathrm{KBr}$ powder led to semitransparent (not completely opaque) pellets when the charge prepared by homogenous dispersion was pressed in a die by a manually operated compressing machine. The pellets were fixed in a dark chamber of the Perkin-Elmer spectrophotometer for recording the spectra in the full infrared range, i.e. in a range $400-4000 \mathrm{~cm}^{-1}$.

\section{Results and Interpretation}

The infrared spectrum of pinacyanol chloride is shown (Figure 2(a)).

The noise which is a peculiar characteristic of a photoconducting medium [21] [22] is clearly observed in electrical conductivity and absorption coefficient in the ranges $3400 \mathrm{~cm}^{-1}$ to $4000 \mathrm{~cm}^{-1}$ and $1650 \mathrm{~cm}^{-1}$ to 2000 $\mathrm{cm}^{-1}$. This is because $\alpha$ and $\sigma$ (conductivity) are related by,

$$
\sigma=\frac{\alpha n_{1} C}{4 \pi}
$$

The higher range noise is due to strong coulomb repulsion among the charge carriers in conduction band. The lower range noise is due to localization near the band edges [23]-[25].

In the mid-frequency range between $1600 \mathrm{~cm}^{-1}$ and $950 \mathrm{~cm}^{-1}$ a background triangular distribution is observed.

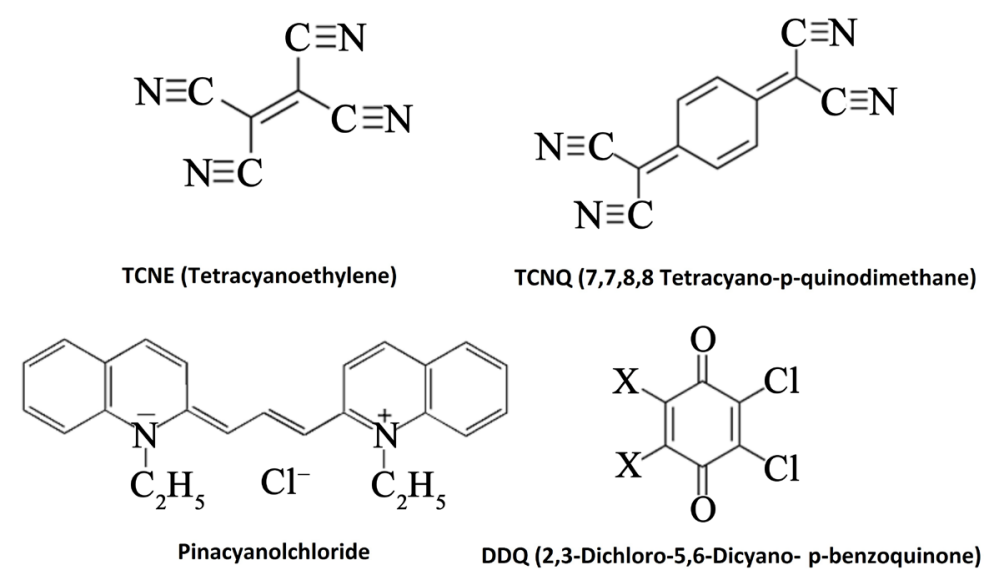

Figure 1. Molecular structures of pinacyanol chloride and organic acceptors. 
The filled density of states for charge carrier tracks a potential barrier. If this barrier is a rectangular barrier, there is very limited transport of charge carriers because the charge carriers need threshold energy for over-barrier hopping. But when such a barrier is triangular due to interaction between chlorine and pinacyanol cation, there is tunneling probability increasing the number of transported charge carriers by three orders of magnitude. Actually this is the origin of photoconductivity of pinacyanol chloride, i.e. $I_{\text {photo }} / I_{\text {dark }}=10^{3}$ as observed earlier.

There is slight increase in transmission below $1000 \mathrm{~cm}^{-1}$ and a plateau is seen below $900 \mathrm{~cm}^{-1}$. At still lower wave numbers below $750 \mathrm{~cm}^{-1}$, a weak asymmetric Gaussian envelope is observed extending down to $400 \mathrm{~cm}^{-1}$ (Figure 2(b)).

This Gaussian band is due to the coupling of rocking, wagging and group vibrations with electronic motion. The band is a characteristic of pinacyanol cation since it is observed in all the CT complexes studied in the present work. The nature of interband transition is studied in the range between $1650 \mathrm{~cm}^{-1}$ and $2000 \mathrm{~cm}^{-1}$ by plotting all possible absorption functions of direct and indirect transitions and deciding the best fit. The comparison of all graphs lead to conclude that the IR band gap is about $0.25 \mathrm{eV}$ and the transition is direct allowed type. Although pinacyanol is a macro molecular substance, the transition is found to be direct rather than indirect because of the molecular symmetry. This shows that a symmetric macromolecule behaves like a small purely organic molecule having negligible dipole moment. The plot of analysis is shown (Figure 2(c)).

The infrared spectrum of (PC-Cl)-chloranil is shown (Figure 3(a)).

Again noise is observed in $3600-3900 \mathrm{~cm}^{-1}$ range. The lower frequency range is spread over a long range compared to PC-Cl above Eg. This is due to disordered molecules of chloranil as compared to fixed lattice of PC-Cl. There are red-shifts of many bands above $E g$ and blue-shifts of many bands below $E g$. This shows that there is softening of high frequency vibrations above $E_{g}$ and anharmonicity in low frequency vibrations below $E_{g}$. The interband transition is allowed direct with a slight lowering of the band gap $(E g-0.22 \mathrm{eV})$ (Figure 3(b)).

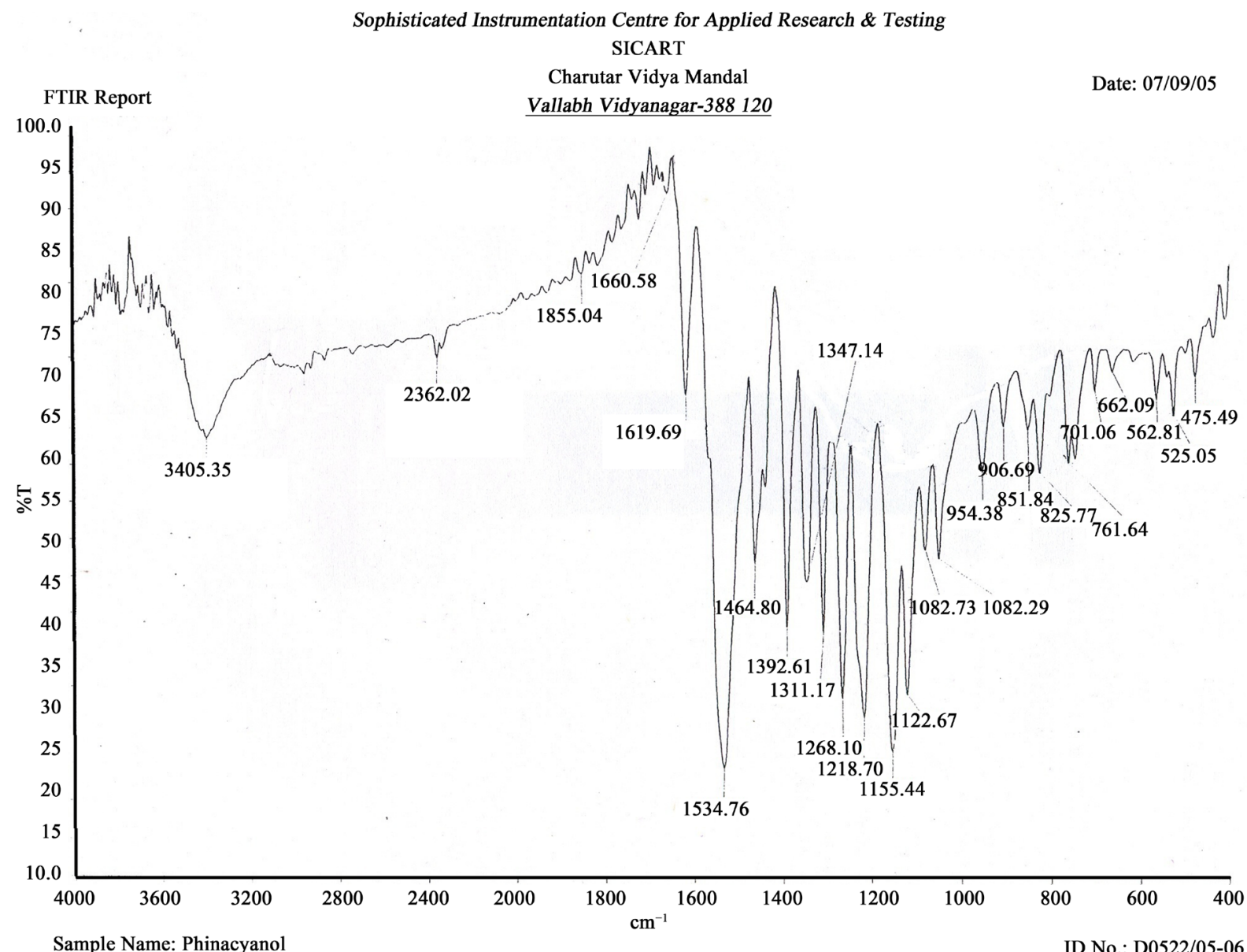

(a) 


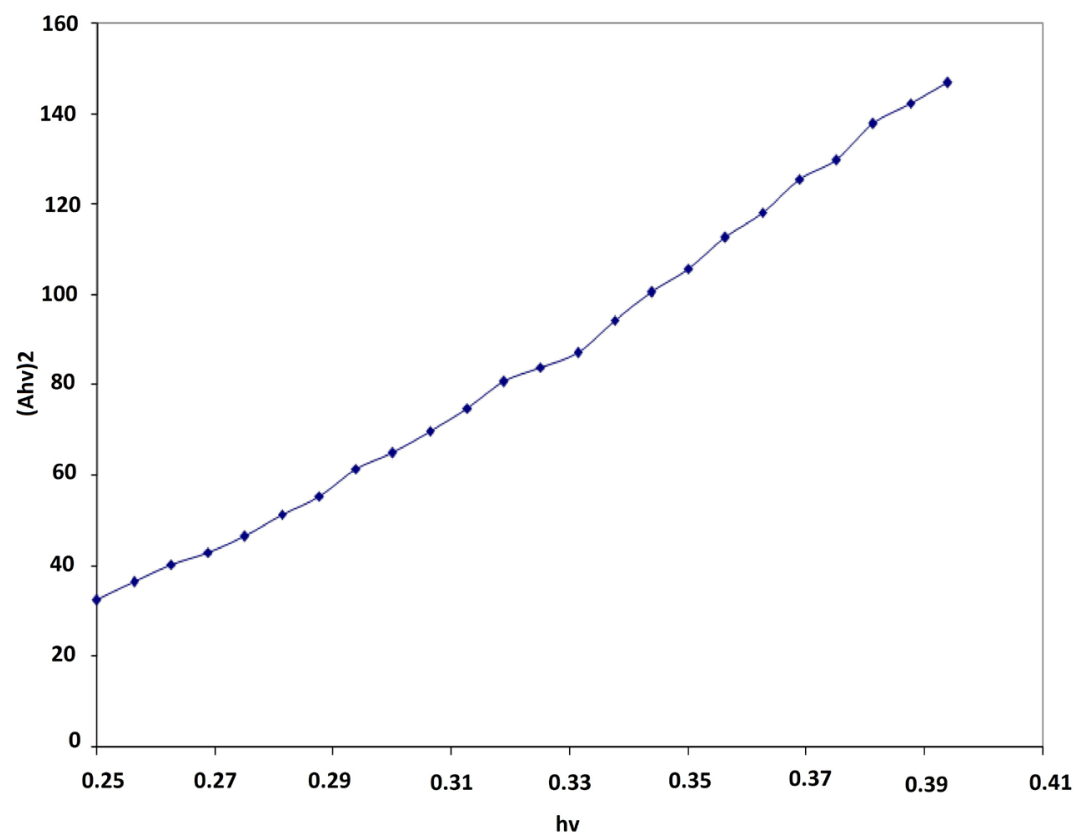

(b)

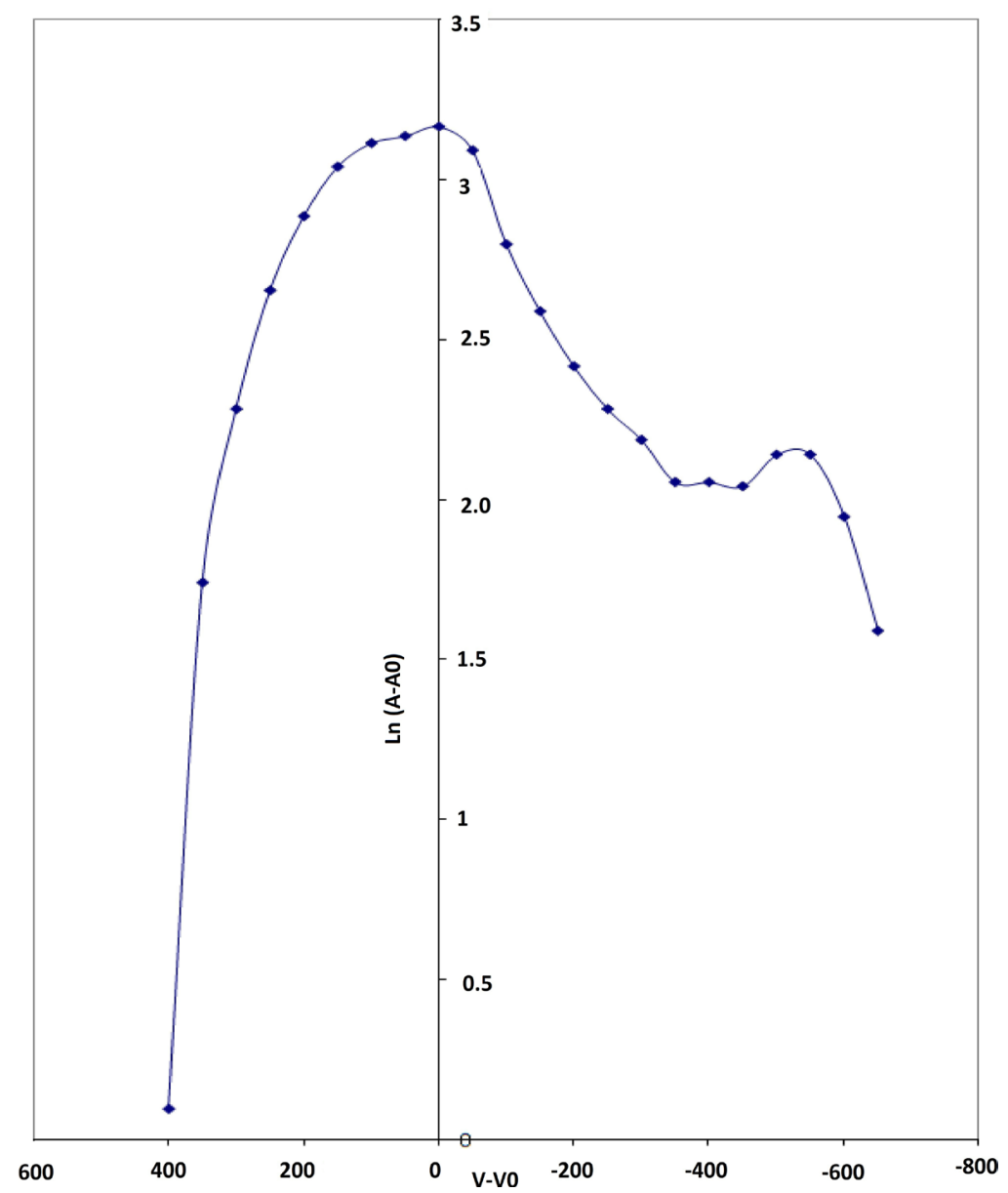

(c)

Figure 2. (a) IR spectrum of pinacyanol chloride; (b) Nature of transition; (c) Low-frequency gaussian. 
Sophisticated Instrumentation Centre for Applied Research \& Testing SICART

Charutar Vidya Mandal

Date: $08 / 10 / 05$

FTIR Report

Vallabh Vidyanagar-388 120

PH.No. (02692) 234966 Fax No. (02692) 238355

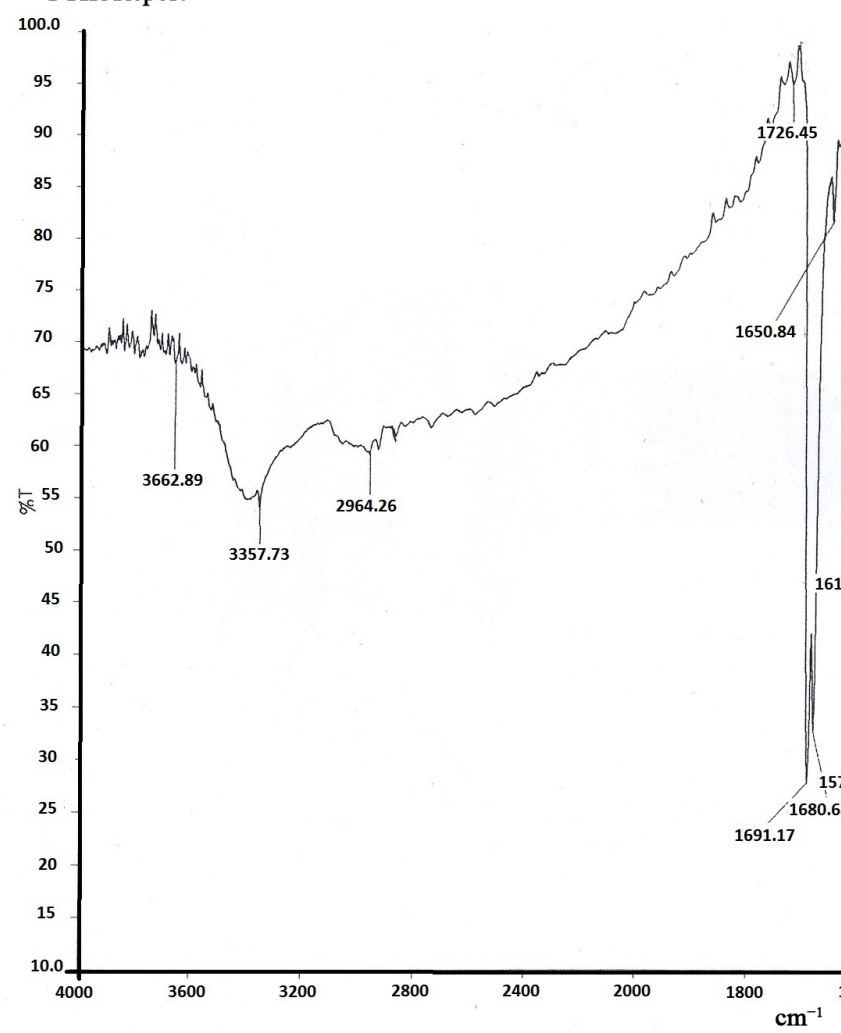

Sample Name: B-3 (PC - Chloranil)

ID NO. : D0688/05-06

(a)

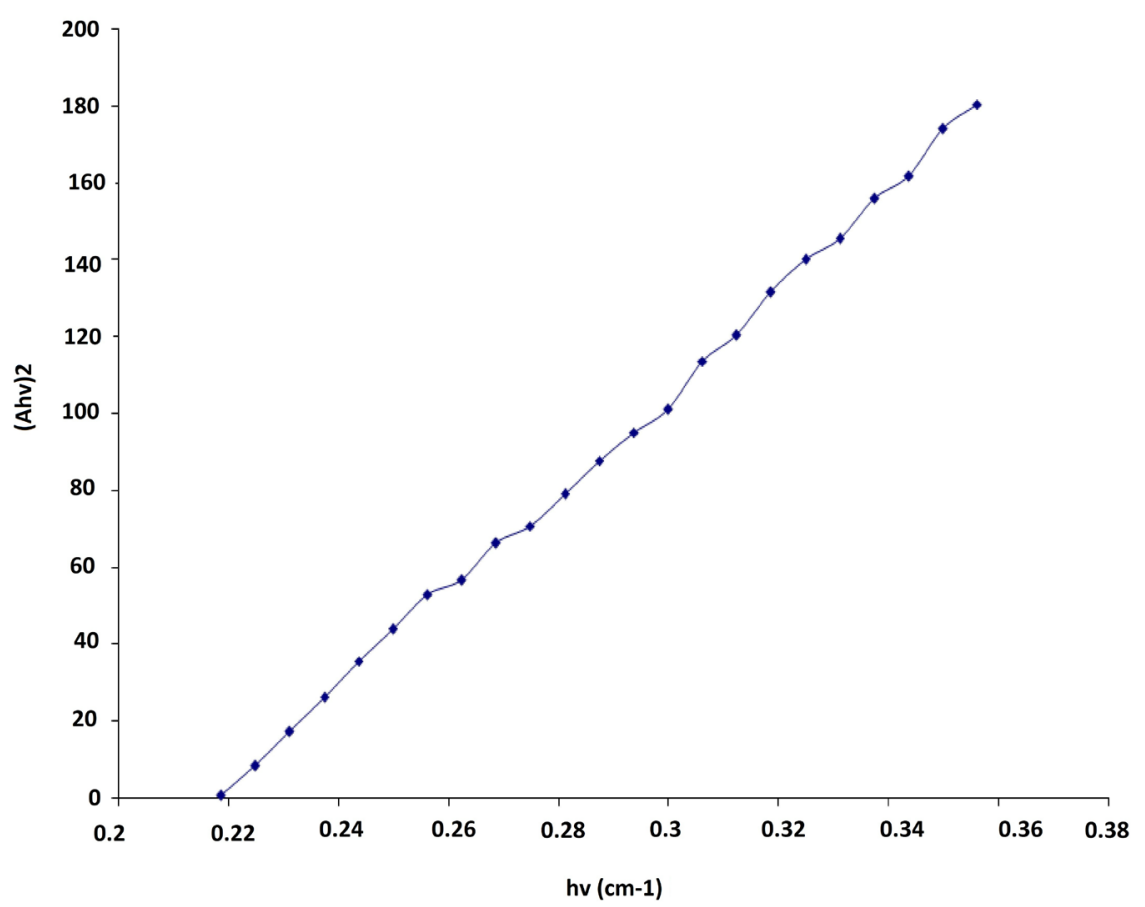

(b) 


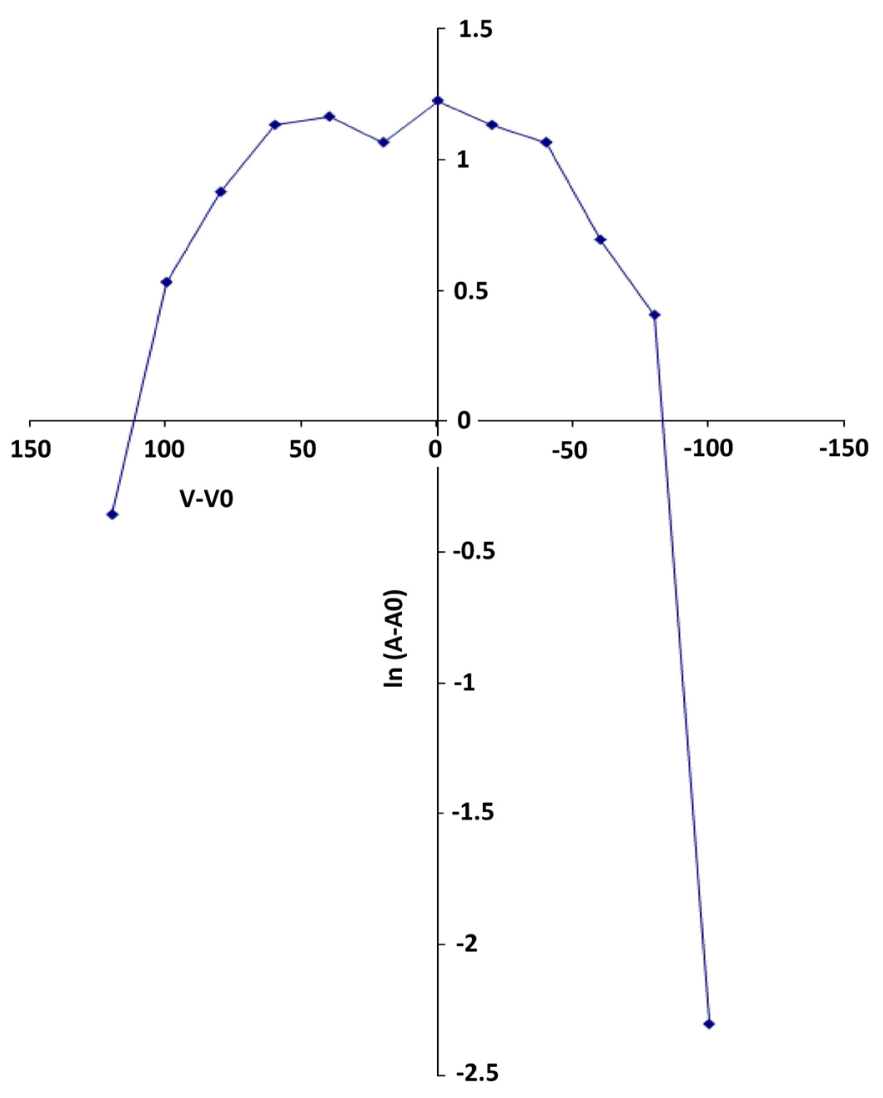

(c)

Figure 3. (a) IR Spectrum of (PC-CL)-chloranil; (b) Nature of transition in (PC-CL)-chloranil; (c) Analysis of gaussian band at low-frequency.

The mid-IR envelope centered around $1150 \mathrm{~cm}^{-1}$ is somewhat distorted from the triangular distribution and has a low-frequency tail. The shape of this envelope can be attributed to imperfect nesting or partial screening. On the other hand, the shape below $1100 \mathrm{~cm}^{-1}$ can be visualized as inverted parabola in transmission, a parabola in absorption coefficient. This can be directly related with band structure. The absorption coefficient and the density of states of charge carriers are proportional. The low-frequency Gaussian curve is preserved but there are no frequency shifts of the bands which provide fine structure. The Gaussian band is almost symmetric. It is more symmetric than same found in PC-Cl alone (Figure 3(c)).

Actually pinacyanol chloride is asymmetric due to $\mathrm{Cl}^{\mathrm{v}}$ attached to the substituent chain on one side as compared to pinacyanol cation. The asymmetry is induced by $\mathrm{Cl}^{\mathrm{v}}$ attached to the substituent chain on one side as compared to pinacyanol cation. The asymmetry is induced by $\mathrm{Cl}^{\mathrm{v}}$ and the net non-zero dipole moment of pinacyanol chloride reduces the second ionization potential of pinacyanol. The organic anion like (chloranil) ${ }^{\mathrm{v}}$ is attracted by (pinacyanol) ${ }^{2+}$ dication due to the quaternization of the second nitrogen atom supporting a second $\mathrm{CH}_{2} \mathrm{CH}_{3}$ group.

The spectrum of (PC-Cl)-DDQ is also shown (Figure4 (a)).

In this case, there is maximum interaction between pinacyanol chloride and DDQ molecule. The DDQ as compared to chloranil easily forms a semi-quinone ion. The interaction is strongly ionic. There is a very large increase in absorption for $h v>E g$. The noise is distributed over a very wide range of absorbed intensity too apart from wide range of frequency or wave number. There is lowering of frequency (wave number) above $E g$ corresponding to softening of lattice vibrations. There is no shift (compared to blue shift in chloranil complex) in the range of mid-IR spectrum below $E g$. This shows absence of anharmonic interaction. The mid-IR envelope is triangular very near to the peak but some kind of imperfect nesting or partial screening dominates for the shape away from the peak. The transition is forbidden direct between $0.25 \mathrm{eV}$ and $0.45 \mathrm{eV}$ with $E g \approx 0.22 \mathrm{eV}$ but having exponential band tailing below $0.25 \mathrm{eV}$. There is band tailing between $0.22 \mathrm{eV}$ and $0.25 \mathrm{eV}$ which is 


\section{Sophisticated Instrumentation Centre for Applied Research \& Testing}

$$
\text { SICART }
$$

Charutar Vidya Mandal

FTIR Report

Vallabh Vidyanagar-388 120

PH.No. (02692) 234966 Fax No. (02692) 238355

$$
100.0
$$
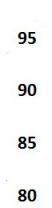

80

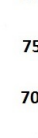

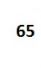

.
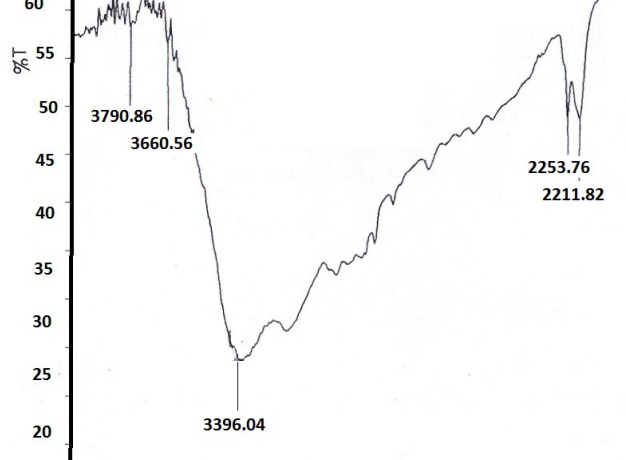

$$
\begin{aligned}
& 45 \\
& 40
\end{aligned}
$$

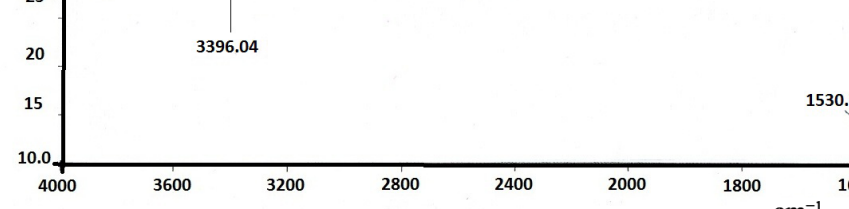

Sample Name: B-1 (Pinacyanol - DDQ)

(a)

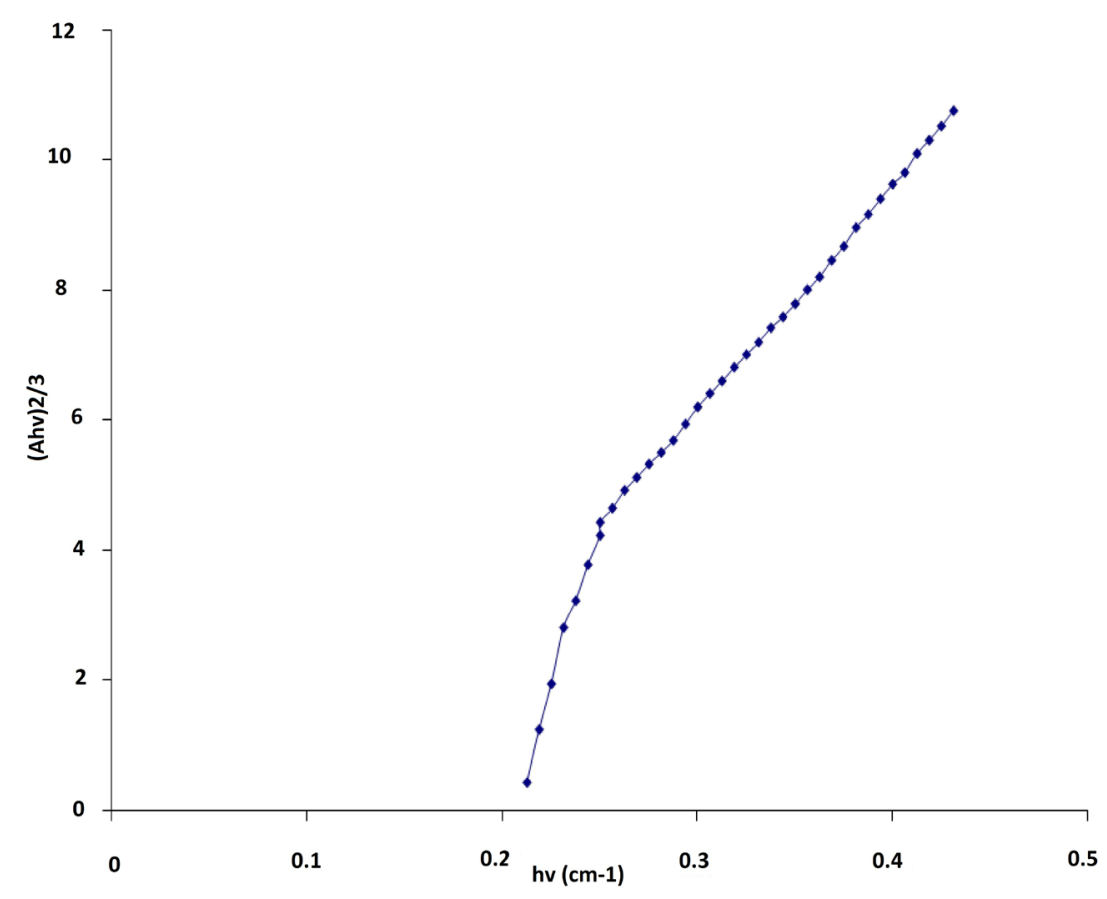

(b) 


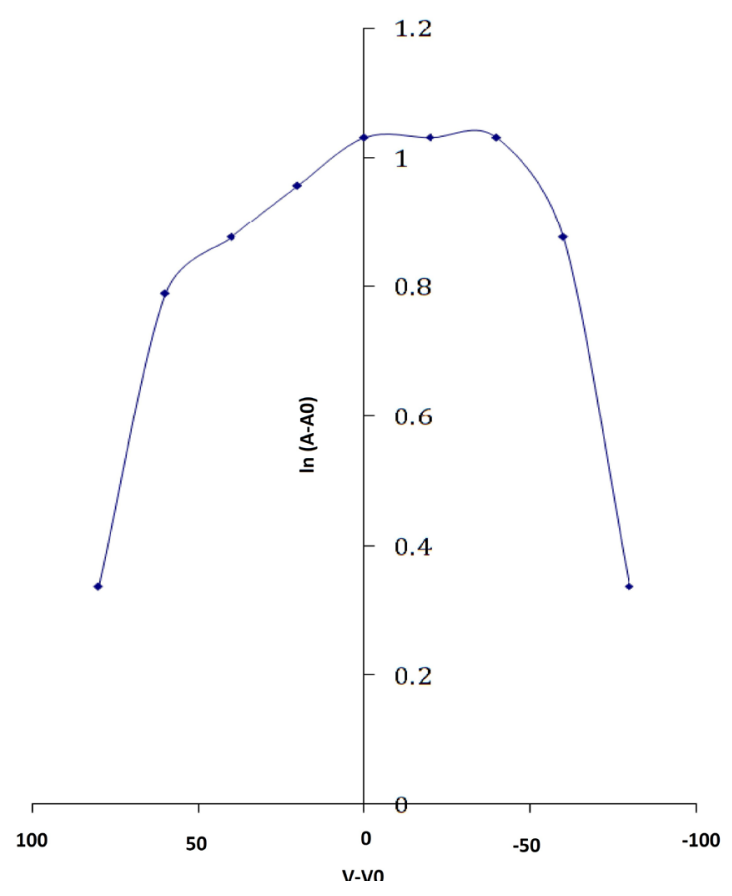

(c)

Figure 4. (a) IR Spectrum of (PC-CL)-DDQ; (b) Nature of transition of (PC-CL)-DDQ; (c) Analysis of gaussian band at low-frequency.

slower function than a step function (Figure 4(b)).

The low frequency Gaussian band around $600 \mathrm{~cm}^{-1}$ is asymmetric showing different slopes of $\ln \left(A-A_{O}\right)$ vs $K-K_{O}$ on both sides (Figure 4(c)). This Gaussian was symmetric in the chloranil complex. The inverted parabola in transmission again is clearly observed below $1100 \mathrm{~cm}^{-1}$. The forbidden direct transition reveals larger intermolecular distance in DDQ complex.

The spectrum of (PC-Cl)-TCNQ is presented in (Figure 5(a)).

The noise in two regions is observed as usual. There are red shifts of the bands above $E g$ associated with softening of lattice vibrations. The monotonous featureless absorption above $1700 \mathrm{~cm}^{-1}$ is analysed for absorption function for interband transition. It is found to be the one describing forbidden direct transition with $E g=0.22$ eV (Figure 5(b)).

The mid-IR envelope is nearly a triangular distribution near a peak around $1100 \mathrm{~cm}^{-1}$. There is slight increase in transmission and a plateau rather than inverted parabola above $1100 \mathrm{~cm}^{-1}$. Weak Gaussian background absorption is found around $550 \mathrm{~cm}^{-1}$ (Figure 5(c)). The Gaussian band is strongly asymmetric.

The last spectrum is that of (PC-Cl)-TCNE (Figure 6(a)). Apart from usual noise in two regions, the nature of transition is found to be forbidden direct with $E g=0.225 \mathrm{eV}$ (Figure 6(b)). The low-frequency Gaussian profile is almost symmetric (Figure 6(c)).

The absorption functions are summarized (Table 1).

The low frequency envelopes (Gaussian bands in all cases) are characterized by the full-width at half- maximum governed by strength of electron-phonon interaction and symmetry or asymmetry (Table 2).

The special mid-IR envelopes related with imperfect nesting or partial screening as well as inverted parabola below $1100 \mathrm{~cm}^{-1}$ are also summarized (Table 3).

The materials prepared in the present work has band gap of the order of $0.22-0.25 \mathrm{eV}$. It is known the total number of absorbed photons has maximum saturation value of $6 \times 10^{17} / \mathrm{sec}-\mathrm{cm}^{2}$ (i.e. per second and per unit area) for $E g \leq 0.5 \mathrm{eV}$ [26] [27]. For $E g>0.5 \mathrm{eV}$, this number drops continuously. Thus the organic semiconductors with $E g<0.5 \mathrm{eV}$ are very good absorbers of photons. This cannot happen in elemental and compound photoconductors, which has $E g>0.5 \mathrm{eV}$. Thus the organic semiconductors can be used as infrared detectors. It is possible to construct other optoelectronic devices such as photodiodes, phototransistors, organic light emitting diodes, etc. using organic semiconductors. 
Sophisticated Instrumentation Centre for Applied Research \& Testing

$$
\text { SICART }
$$

Charutar Vidya Mandal

FTIR Report

Vallabh Vidyanagar-388 120

PH.No. (02692) 234966 Fax No. (02692) 238355

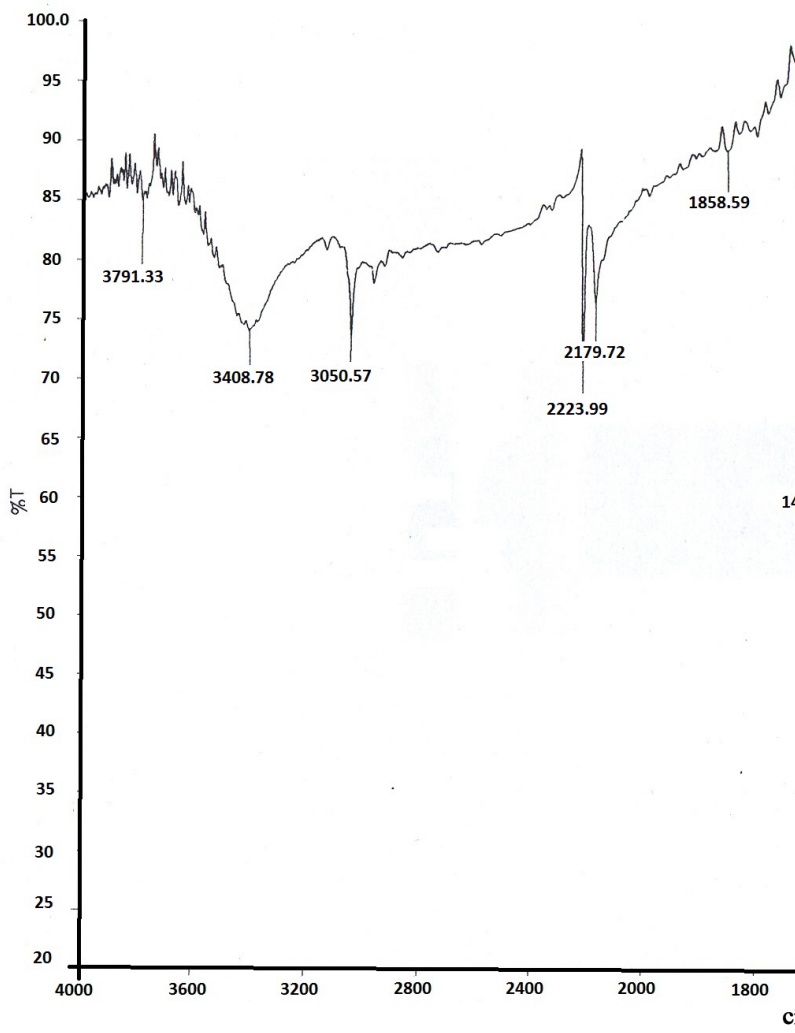

Sample Name: B-4 (PC - TCNQ)

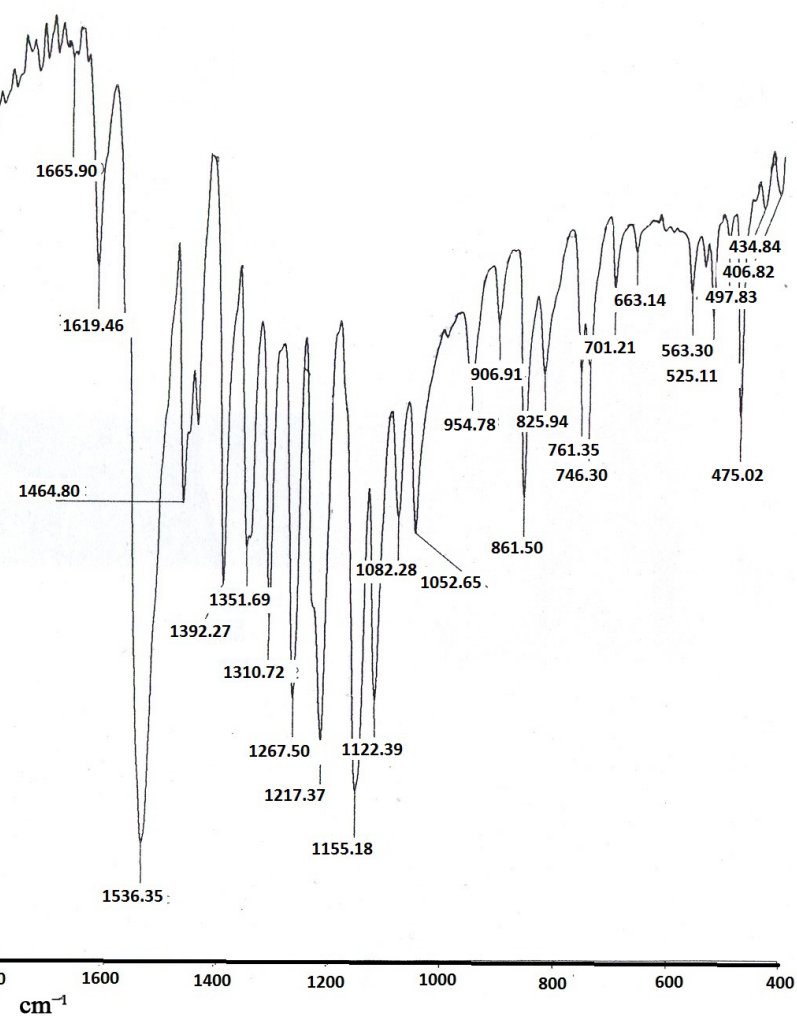

(a)

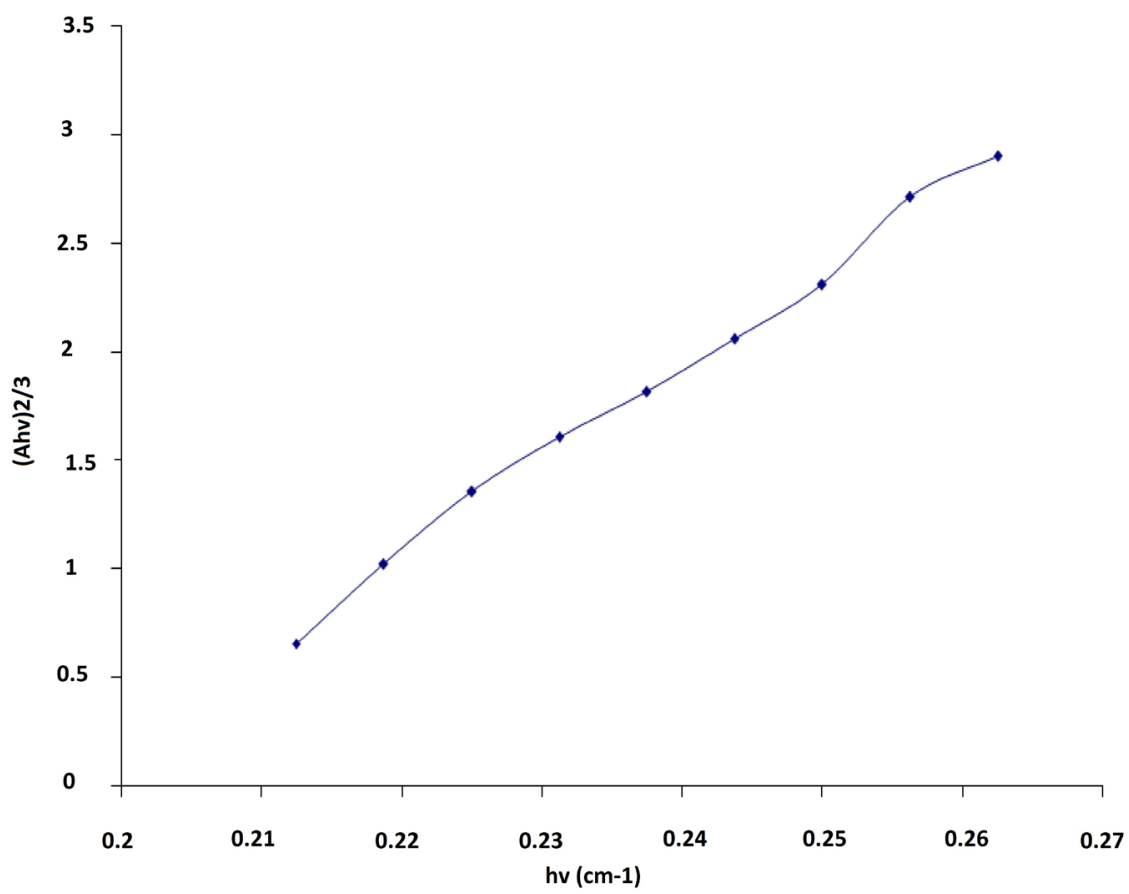

(b) 


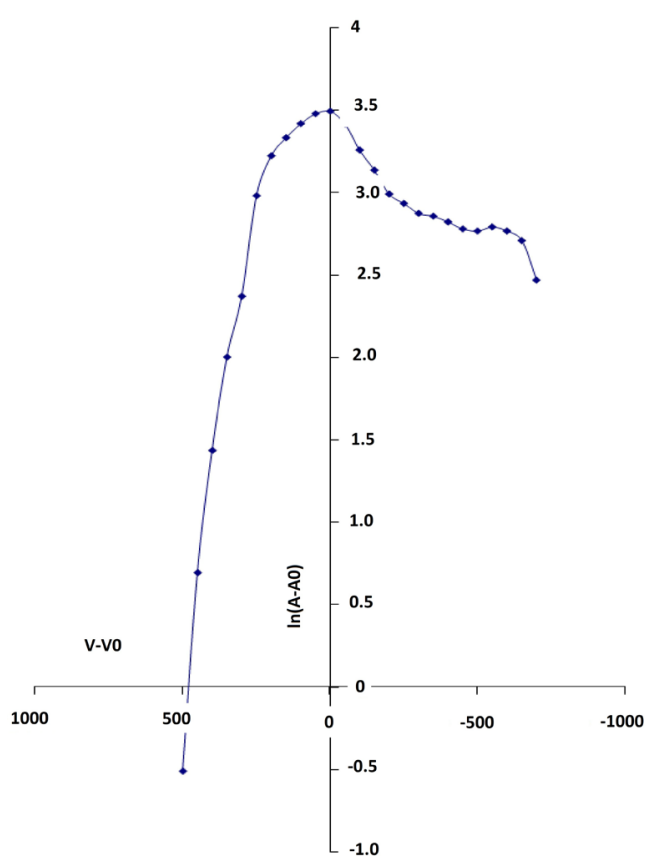

(c)

Figure 5. (a) IR spectrum of (PC-CL)-TCNQ; (b) Nature of transition in (PC-CL)-TCNQ; (c) Analysis of Gaussian at low-frequency.

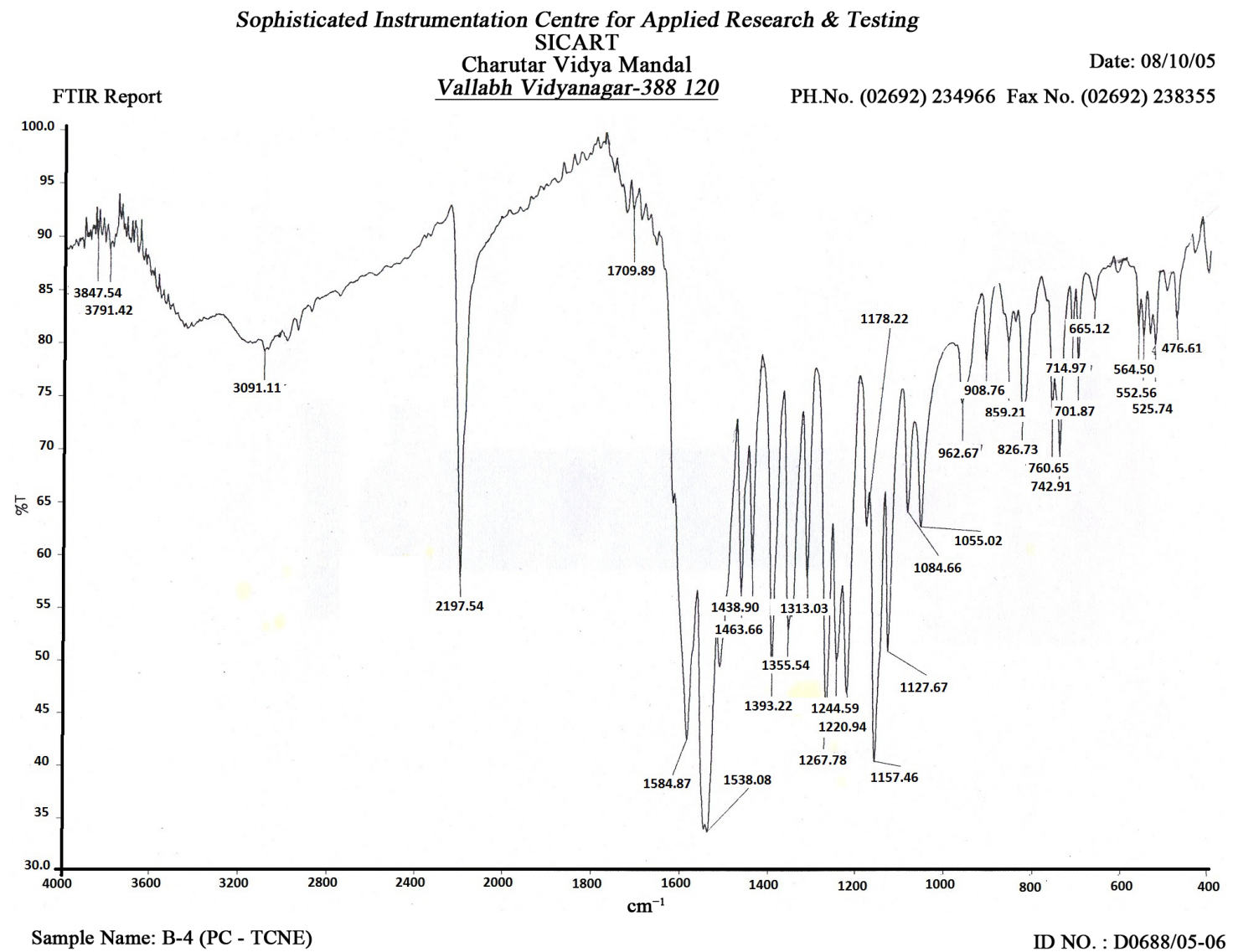

(a) 


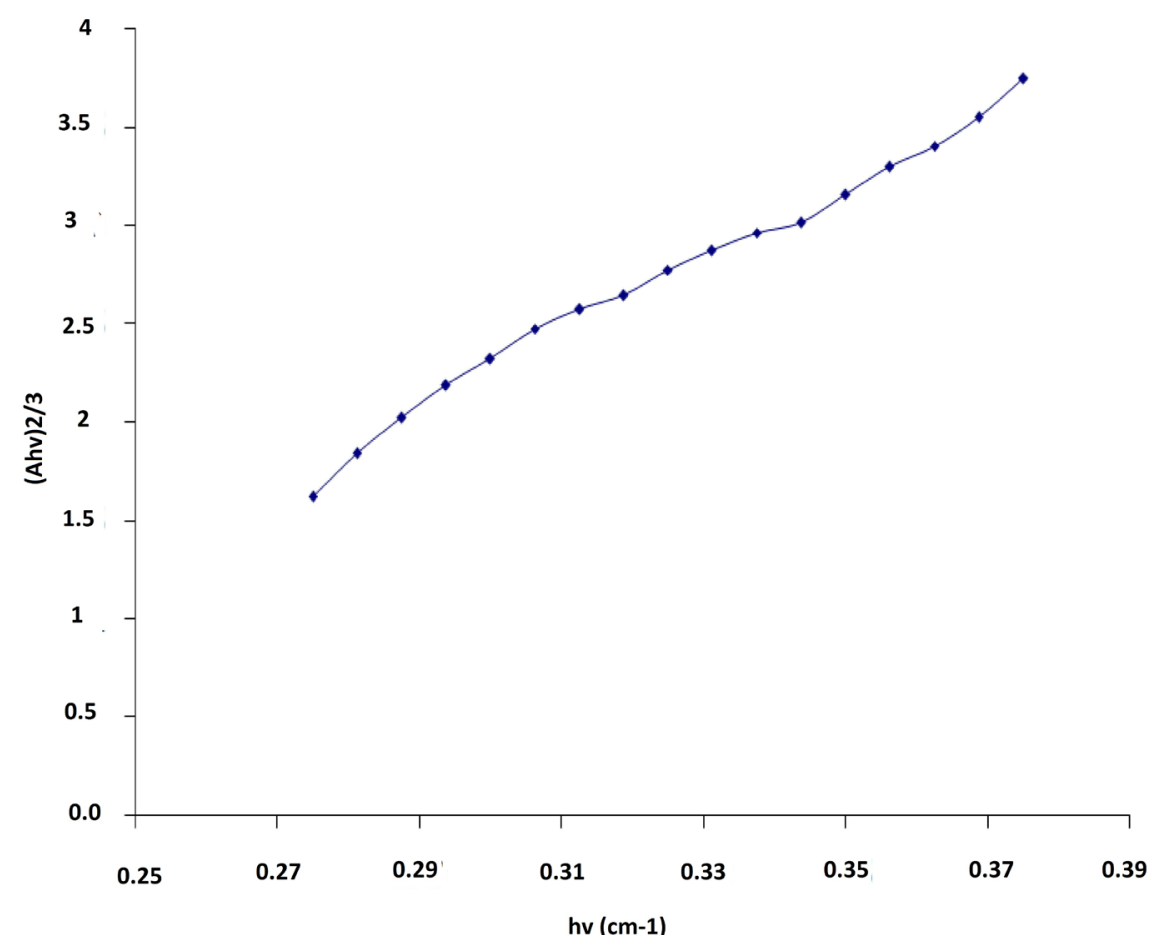

(b)

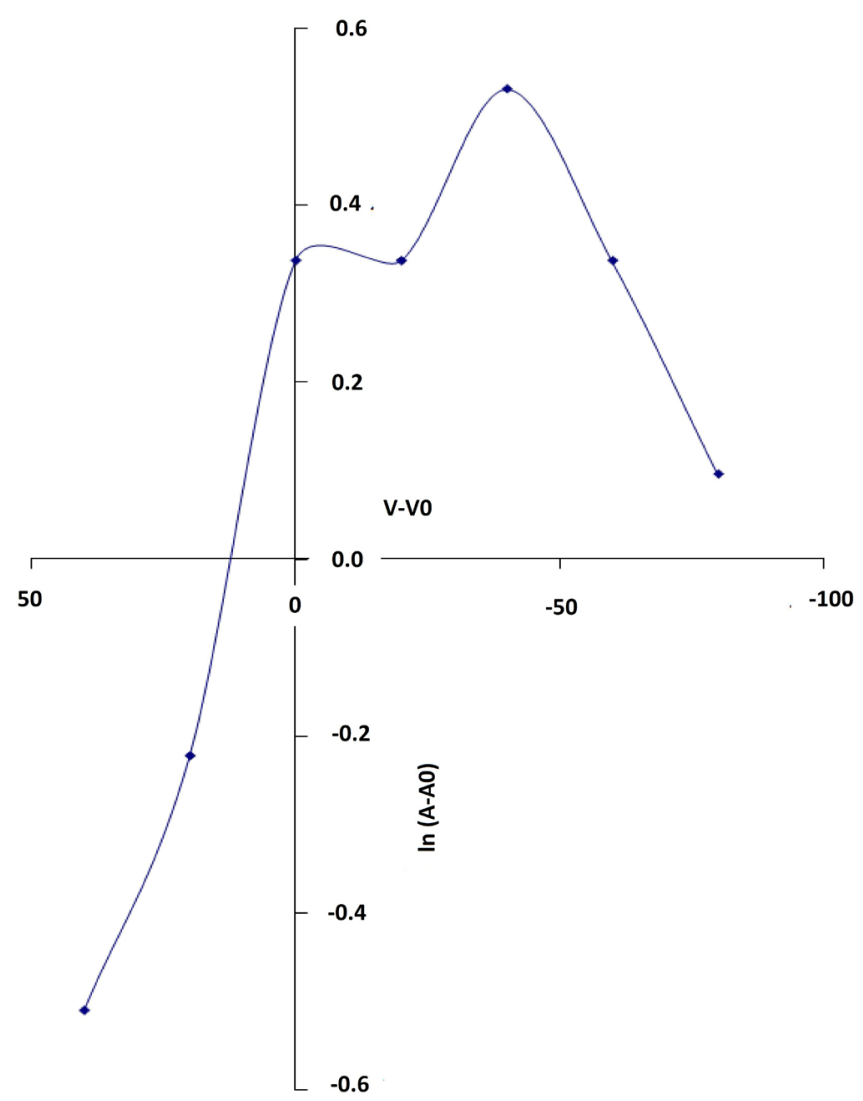

(c)

Figure 6. (a) IR spectrum of (PC-CL)-TCNE; (b) Nature of transition in (PC-CL)-TNCE; (c) Analysis of Gaussian at low-frequency. 
Table 1. Nature of transition in pinacyanol chloride and its charge transfer complexes.

\begin{tabular}{|c|c|c|c|}
\hline Compound & Absorption Function and $E g(\mathrm{eV})$ & Nature of Transition & Range of Absorption Function $\left(\mathrm{cm}^{-1}\right)$ \\
\hline Pinacyanol chloride & $\begin{array}{l}\alpha h v=A\left(h v-E_{g}\right)^{1 / 2} \\
E_{g}=0.25 \mathrm{eV}\end{array}$ & $\begin{array}{l}\text { Direct allowed } \\
\text { Transition }\end{array}$ & $1650-3000$ \\
\hline $\begin{array}{l}\text { Pinacyanol chloranil } \\
\text { (PC-Cl) }\end{array}$ & $\begin{array}{l}\alpha h v=A\left(h v-E_{g}\right)^{1 / 2} \\
E_{g}=0.22 \mathrm{eV}\end{array}$ & $\begin{array}{l}\text { Direct allowed } \\
\text { Transition }\end{array}$ & $1750-3000$ \\
\hline (PC-Cl)-DDQ & $\begin{array}{l}\alpha h v=A\left(h v-E_{g}\right)^{3 / 2} \\
E_{g}=0.21 \mathrm{eV}\end{array}$ & $\begin{array}{l}\text { Direct forbidden } \\
\text { Transition }\end{array}$ & $1670-3000$ \\
\hline (PC-Cl)-TCNQ & $\begin{array}{l}\alpha h v=A\left(h v-E_{g}\right)^{3 / 2} \\
E_{g}=0.21 \mathrm{eV}\end{array}$ & $\begin{array}{l}\text { Direct forbidden } \\
\text { Transition }\end{array}$ & $1700-2200$ \\
\hline (PC-Cl)-TCNE & $\begin{array}{l}\alpha h v=A\left(h v-E_{g}\right)^{3 / 2} \\
E_{g}=0.225 \mathrm{eV}\end{array}$ & $\begin{array}{l}\text { Direct forbidden } \\
\text { Transition }\end{array}$ & $1750-3100$ \\
\hline
\end{tabular}

Table 2. Low-frequency envelope in pinacyanol chloride and its charge transfer complexes.

\begin{tabular}{cccc}
\hline Compound & Exact Line shape & $\begin{array}{c}\text { Full width at half maximum } \\
\left(\mathbf{c m}^{-\mathbf{1}} \text { and eV) (FWHM) }\right.\end{array}$ & Range (cm $\left.^{-\mathbf{1}}\right)$ \\
\hline Pinacyanol chloride & Asymmetric Gaussian & $200(0.025)$ & $400-800$ \\
Pinacyanol Chloranil (PC-Cl) & Centrally split symmetric Gaussian & $250(0.032)$ & $400-750$ \\
(PC-Cl)-DDQ & Centrally split asymmetric Gaussian & $300(0.0375)$ & $400-800$ \\
(PC-Cl)-TCNQ & Asymmetric Gaussian & $270(0.034$ & $400-700$ \\
(PC-Cl)-TCNE & Centrally split asymmetric Gaussian & $120(0.015)$ & $420-620$ \\
\hline
\end{tabular}

Table 3. Special mid-IR envelope related with imperfect nesting or screening.

\begin{tabular}{|c|c|c|c|}
\hline Compound & Shape of the envelope & Range of the envelope $\left(\mathrm{cm}^{-1}\right)$ & Shape of the curve below $1100\left(\mathrm{~cm}^{-1}\right)$ \\
\hline Pinacyanol chloride & Symmetric triangular distribution & $650-1650$ & Indirect transition \\
\hline $\begin{array}{c}\text { Pinacyanol chloranil } \\
\text { (PC-Cl) }\end{array}$ & $\begin{array}{l}\text { Imperfect nesting } \\
\text { for cosinusoidal gap }\end{array}$ & $650-1700$ & Inverted parabola \\
\hline (PC-Cl)-DDQ & Imperfect nesting for cosinusoidal gap & $700-1700$ & Inverted parabola \\
\hline (PC-Cl)-TCNQ & Imperfect nesting for cosinusoidal gap & $800-1700$ & Indirect transition \\
\hline (PC-Cl)-TCNE & Not observed & - & Monotonous increase and plateau \\
\hline
\end{tabular}

\section{Conclusions}

There are two types of organic photoconductors according to the infrared spectroscopy. In one case, transmission is large and constant above $E g$ and in the other case, absorption builds up above $E g$ following direct or indirect transition. In CTCs of Pinacyanol Chloride (PC), the band gap is reduced by $0.03 \mathrm{eV}$ to $0.04 \mathrm{eV}$, but transition remaining direct type. The mid-IR envelope neither Lorentzian nor Gaussian is triangular type as determined by imperfect nesting and partial screening. Only at low-frequency below $800 \mathrm{~cm}^{-1}$, there is asymmetric Gaussian band in PC which becomes centrally-split Gaussian band in Chloranil, DDQ and TCNE complexes. The central splitting in Gaussian may be arising from density of states or from Davydov-type splitting.

Pinacyanol chloride reveals tunneling of charge carriers through a triangular barrier between $1700 \mathrm{~cm}^{-1}$ and $700 \mathrm{~cm}^{-1}$ arising from imperfect nesting or triangular barrier between valence band and conduction band associated with internal Franz-Keldysh effect (Redfield effect). The material has direct band gap and shows allowed transition. Band gap is somewhat reduced in the four CT complexes. Mid-IR spectrum shows absorption function arising from imperfect nesting or partial screening which is never observed in either binary or ternary CT complexes. 


\section{Acknowledgements}

Authors acknowledge Department of Physics, Sardar Patel University, Vallabh Vidyanagar, for supporting the work by way of extending laboratory facility, chemicals, instruments and sample characterization charges. Authors are also thankful to management of Gujarat Energy Research \& Management Institute (GERMI) for providing necessary permissions to carry out the work at Physics Department and constant encouragement.

\section{References}

[1] Foster, R. (1969) Organic Charge Transfer Complexes. Academic Press, London-New York.

[2] Gutman, F. and Lyons, L.E. (1967) Organic Semiconductors. John Wiley \& Sons, Inc., Hoboken, 776.

[3] Goodings, E.P. (1976) Conductivity and Superconductivity in Polymers. Chemical Society Reviews, 5, 95-123. http://dx.doi.org/10.1039/cs9760500095

[4] Miller, J.S. and Epstein, A.J. (1976) One-Dimensional Inorganic Complexes. In: Lippard, S.J., Ed., Progress in Inorganic Chemistry, Vol. 20, An Interscience Publication, John Wiley \& Sons, Inc., Hoboken.

[5] Garito, A.F. and Heeger, A.J. (1974) Design and Synthesis of Organic Metals. Accounts of Chemical Research, 7, 232240. http://dx.doi.org/10.1021/ar50079a004

[6] Ehara, S., Takagi, T., Yoshida, T., Imaba, H., Naito, H. and Okuda, M. (1991) Mod. Phys. Letters B,C Singapore, 6, 1205.

[7] Singh, R.A., Singh, R. and Verma, S.M. (1991) Molecular Semiconduction in Some Substituted Phenothiazine-2,3Dichloro-5,6-Dicyano-p-Benzoquinone Charge-Transfer Complexes. Indian Journal of Technology, 29, 241.

[8] Pal, P. and Misra, T.N. (1991) Indian Journal of Physics, 65 B, 459.

[9] Gunster, S., Siebentrost, S., Elbe, J., Kreienhoop, L., Tennigkeit, B., Wohrle, D., Memming, R. and Meissner, D. (1992) Investigations of Porphyrins and Aromatic Tetracarboxylic Acid Diimides for Use in Photovoltaics. Molecular Crystals and Liquid Crystals, 218, 117-122. http://dx.doi.org/10.1080/10587259208047026

[10] Fattori, V., Dimarco, P., Giro, G. and Kqlinowaski, J. (1992) mol. Cryst. Liq-cryst., 211, 313.

[11] Sen, S., Pal., P. and Mistra, T.N. (1993) Semiconduction Properties of Some Polyene-Iodine Charge-Transfer Complexes and Their Application in Solid-State Batteries. Journal of Materials Science, 28, 1367-1371. http://dx.doi.org/10.1007/BF01191979

[12] Lefdil, A.M., Sayah, D. and Cadens, M. (1992) Oriented Polyacetylene (p)-Cadmium Sulphide (n) Heterojunctions. Journal of Materials Science Letters, 11, 160-161. http://dx.doi.org/10.1007/BF00724678

[13] Hamann, C. and Mrwa, A. (1992) Switching Behaviour of Lead Phthalocyanine Thin Films. International Journal of Electronics, 73, 1039-1040. http://dx.doi.org/10.1080/00207219208925762

[14] Shafai, T.S. and Gould, R.D. (1992) Electrical Characteristics of Lead Phthalocyanine Thin Films Using Aluminium Schottky Barriers and Gold Ohmic. International Journal of Electronics, 73, 1043-1045. http://dx.doi.org/10.1080/00207219208925764

[15] Antohe, S. (1992) Rev. Mod. Phys., 37, 309.

[16] Nazarova, I.B., Krinichnyl, V.I. and Greenberg, L.M. (1993) Schottky Diodes Based on Poly(p-phenylene) and Poly(1,4-dipyrrolobenzene). Synthetic Metals, 53, 399-402. http://dx.doi.org/10.1016/0379-6779(93)91108-E

[17] Fichou, D., Horowitz, G. and Garnier, F. (1992) Extended Oligothiophenes: New Materials for Molecular Electronics. Molecular Crystals and Liquid Crystals, 217, 193-196. http://dx.doi.org/10.1080/10587259208046899

[18] Scott, J.C., Pautmeir, L.T. and Moerner, W.E. (1992) Photoconductivity studies of photorefractive polymers. Journal of the Optical Society of America B, 9, 2059-2064.

[19] Pfleger, J., Kminek, I. and Nešpůrek, S. (1992) Photoelectrical Properties of High Density Polyacetylene. Synthetic Metals, 46, 189-199. http://dx.doi.org/10.1016/0379-6779(92)90342-G

[20] Yang, Y., Lee, J.Y., Jain, A.K., Kumar, J., Tripathy, S.K., Matsuda, H., Okada, S. and Nakanishi, H. (1991) Polarization-Dependent Photocurrent in Thin Film Polydiacetylene Single Crystals. Journal of Physics: Condensed Matter, 3, 9563-9568.

[21] van der Ziel, A. (1971) Solid State Physical Electronics. 2nd Edition, Prentice-Hall of India, Pvt. Ltd., New Delhi, 206.

[22] Bhattarcharya, P. (2002) Semiconductor Optoelectronic Devices. 2nd Edition, Prentice-Hall of India Private Limited, New Delhi, 344.

[23] Banyai, L. (1964) Physique des Semiconductors. Dunod, Paris, 417. 
[24] Gubanov, A.I. (1965) Quantum Electron Theory of Amorphous Semiconductors. Consultants Bureau, New York.

[25] Oza, A.T. (1982) Electrical Resistivity of $\alpha$-Cyclodextrin-KI-I ${ }_{2} \cdot 4 \mathrm{H}_{2} \mathrm{O}$ Crystals. Physica Status Solidi (b), 114, K171K176. http://dx.doi.org/10.1002/pssb.2221140273

[26] Angrist Stanley, W. (1971) Direct Energy Conversion. 2nd Edition, Allyn and Bacon Inc., Boston, 192.

[27] Culp Archie Jr., W. (1987) Principles of Energy Conversion. McGraw-Hill Book Company, New Delhi, 373. 